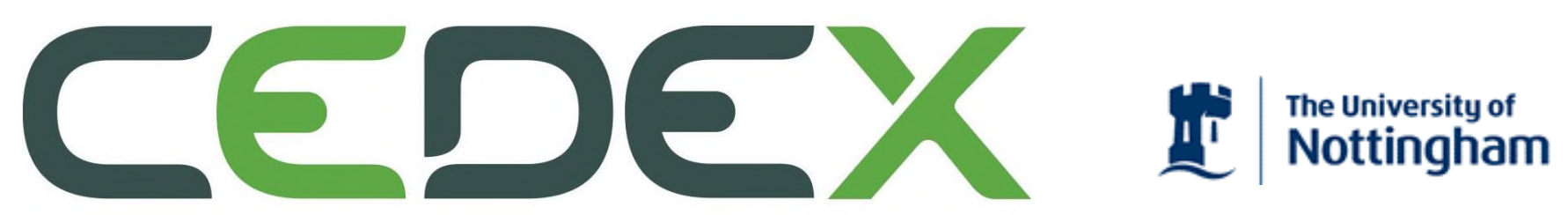

CENTRE FOR DECISION RESEARCH \& EXPERIMENTAL ECONOMICS

Discussion Paper No. 2012-11

J. Arin, V. Feltkamp and M. Montero July 2012
Coalitional Games with Veto Players: Myopic and Rational Behavior 

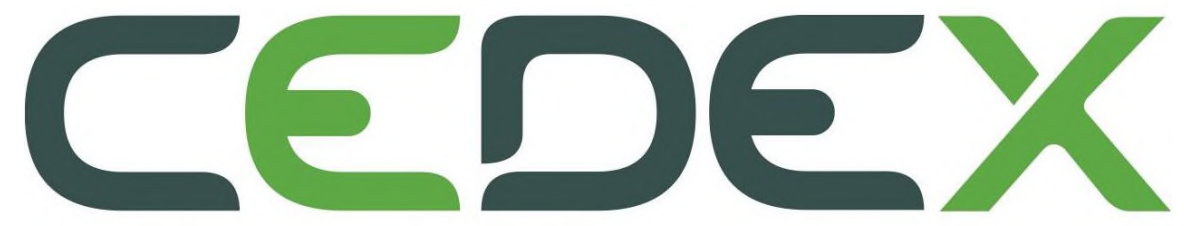

CENTRE FOR DECISION RESEARCH \& EXPERIMENTAL ECONOMICS

The Centre for Decision Research and Experimental Economics was founded in 2000, and is based in the School of Economics at the University of Nottingham.

The focus for the Centre is research into individual and strategic decision-making using a combination of theoretical and experimental methods. On the theory side, members of the Centre investigate individual choice under uncertainty, cooperative and non-cooperative game theory, as well as theories of psychology, bounded rationality and evolutionary game theory. Members of the Centre have applied experimental methods in the fields of public economics, individual choice under risk and uncertainty, strategic interaction, and the performance of auctions, markets and other economic institutions. Much of the Centre's research involves collaborative projects with researchers from other departments in the UK and overseas.

Please visit http://www.nottingham.ac.uk/cedex for more information about the Centre or contact

Sue MacCormick

Centre for Decision Research and Experimental Economics

School of Economics

University of Nottingham

University Park

Nottingham

NG7 2RD

Tel: +44 (0)1159515620

Fax: +44 (0) 1159514159

sue.maccormick@nottingham.ac.uk

The full list of CeDEx Discussion Papers is available at

http://www.nottingham.ac.uk/cedex/publications/discussion-papers 


\title{
Coalitional games with veto players: myopic and rational behavior
}

\author{
J. Arin, V. Feltkamp ${ }^{\dagger}$ and M. Montero
}

July 18, 2012

\begin{abstract}
This paper studies a noncooperative allocation procedure for coalitional games with veto players. The procedure is similar to the one presented by Dagan et al. (1997) for bankruptcy problems. According to it, a player, the proposer, makes a proposal that the remaining players must accept or reject. We present a model where the proposer can make sequential proposals over $n$ periods. If responders are myopic maximizers (i.e. consider each period in isolation), the only subgame perfect equilibrium outcome is the serial rule of Arin and Feltkamp (2012) regardless of the order of moves. If all players are rational, the serial rule still arises as the unique subgame perfect equilibrium outcome if the order of moves is such that stronger players must respond to the proposal after weaker ones.
\end{abstract}

Keywords: veto players, noncooperative bargaining, myopic behavior, serial rule.

JEL classification: C71, C72, C78.

*Dpto. Ftos. A. Económico I, University of the Basque Country, L. Agirre 83, 48015 Bilbao, Spain. Email: franciscojavier.arin@ehu.es.

$\dagger$ Maastricht School of Management, PO Box 1203, 6201 BE Maastricht, The Netherlands. Email: Feltkamp@msm.nl.

${ }^{\ddagger}$ Corresponding author. School of Economics, University of Nottingham, Nottingham NG7 2RD, United Kingdom. Phone: +44 115 9515468. Fax: +44 115 9514159. Email: maria.montero@nottingham.ac.uk. 


\section{Introduction}

Dagan et al. (1997) introduced a noncooperative bargaining procedure for bankruptcy problems. In this procedure the player with the highest claim has a distinguished role. He makes a proposal and the remaining players accept or reject sequentially. Players who accept the proposal leave the game with their share; if a player rejects the proposal this conflict is solved bilaterally by applying a normative solution concept (a "bilateral principle" based on a bankruptcy rule) to a two-claimant bankruptcy problem in which the estate is the sum of the two proposed payoffs. They show that a large class of consistent and monotone bankruptcy rules can be obtained as the Nash outcomes of the game. They describe this kind of procedure as consistency based: starting from a consistent solution concept, they construct extensive forms whose subgames relate to the respective reduced cooperative games and by finding the equilibrium of the extensive form they are able to provide noncooperative foundations for the consistent solution of interest.

Arin and Feltkamp (2007) consider an analogous procedure for veto balanced games, in which a veto player is the proposer and the bilateral principle in the event of a disagreement is the standard solution of a Davis-Maschler reduced game (unless this would result in a negative payoff for the responder, in which case the responder gets zero). The nucleolus is a natural candidate to be a Nash outcome in this case, since it satisfies the Davis-Maschler reduced game property. However, it is not always obtained because it does not satisfy aggregate monotonicity even in the class of veto balanced games: if the veto balanced game $v$ is such that reducing $v(N)$ never increases the nucleolus payoff for the veto player, the nucleolus is a Nash outcome; otherwise the proposer is better-off by making an inefficient proposal rather than proposing the nucleolus.

In the present paper, we modify the above procedure by allowing the proposer to make a fixed number of sequential proposals, so that players can continue bargaining over the remainder if the first proposal did not exhaust the value of the grand coalition. Each period results in a partial agreement, and then a new TU game is formed where the values of the coalitions take into account the agreements reached so far; the final outcome is the sum 
of all partial agreements. We focus on the case in which the proposer can make as many proposals as there are players in the game. We then analyze the game assuming myopic behavior of the responders, that is, we assume that responders consider each period in isolation, accepting or rejecting the current proposal without anticipating the effects of their decision on future periods. The proposer is assumed to behave rationally, taking into account the effect of his actions on future periods and also taking into account that the responders behave myopically. We refer to this kind of strategy profile as a myopic best response equilibrium (MBRE).

It turns out that all MBRE lead to the same outcome, which is the serial rule of Arin and Feltkamp (2012). This solution concept is based on the idea that the strength of player $i$ can be measured by the maximum amount a coalition can obtain without player $i$, denoted by $d_{i}$. Since it is impossible for any coalition to obtain a payoff above $d_{i}$ without $i$ 's cooperation, player $i$ can be viewed as having a veto right over $v(N)-d_{i}$. The serial rule divides $v(N)$ in segments, and each segment is equally divided between the players that have a veto right over it. The serial rule is always in the core, and unlike the nucleolus it satisfies aggregate monotonicity.

If responders take into account the effect of their actions on future periods, there may be subgame perfect equilibrium outcomes different from the serial rule. The order of moves may be such that the proposer is able to hide some payoff from a stronger player with the cooperation of a weaker player: the proposal faced by the stronger player is not too favorable for the proposer so that the stronger player cannot challenge it, but later on a weak player rejects the proposal and transfers some payoff to the proposer; the weak player may have an incentive to do so because of the effect of this agreement on future periods. However, if the order of moves is such that stronger players have the last word in the sense that they respond to the proposal after weaker ones, the only subgame perfect equilibrium outcome is the serial rule. Hence, myopic and rational behavior of the responders lead to the same outcome in this case. 


\section{Preliminaries}

\section{$2.1 \quad$ TU games}

A cooperative n-person game in characteristic function form is a pair $(N, v)$, where $N$ is a finite set of $n$ elements and $v: 2^{N} \rightarrow \mathbb{R}$ is a real-valued function on the family $2^{N}$ of all subsets of $N$ with $v(\emptyset)=0$. Elements of $N$ are called players and the real-valued function $v$ the characteristic function of the game. We shall often identify the game $(N, v)$ with its characteristic function and write $v$ instead of $(N, v)$. Any subset $S$ of the player set $N$ is called a coalition. The number of players in a coalition $S$ is denoted by $|S|$. In this work we only consider games where all coalitions have nonnegative worth.

A payoff allocation is represented by a vector $x \in \mathbb{R}^{n}$, where $x_{i}$ is the payoff assigned by $x$ to player $i$. We denote $\sum_{i \in S} x_{i}$ by $x(S)$. If $x(N) \leq v(N)$, $x$ is called a feasible allocation; if $x(N)=v(N), x$ is called an efficient allocation. An efficient allocation satisfying $x_{i} \geq v(i)$ for all $i \in N$ is called an imputation and the set of imputations is denoted by $I(N, v)$. The set of nonnegative feasible allocations is denoted by $D(N, v)$ and formally defined as follows

$$
D(N, v)=\left\{x \in \mathbb{R}^{N}: x(N) \leq v(N) \text { and } x_{i} \geq 0 \text { for all } i \in N\right\} .
$$

The core of a game is the set of imputations that cannot be blocked by any coalition, i.e.

$$
C(N, v)=\{x \in I(v): x(S) \geq v(S) \text { for all } S \subseteq N\} .
$$

A game with a nonempty core is called a balanced game. A game $v$ is a veto-rich game if it has at least one veto player and the set of imputations is nonempty. A player $i$ is a veto player if $v(S)=0$ for all coalitions where player $i$ is not present. A balanced game with at least one veto player is called a veto balanced game.

A solution $\phi$ on a class of games $\Gamma_{0}$ is a correspondence that associates with every game $(N, v)$ in $\Gamma_{0}$ a set $\phi(N, v)$ in $\mathbb{R}^{N}$ such that $x(N) \leq v(N)$ for 
all $x \in \phi(N, v)$. This solution is called efficient if this inequality holds with equality. The solution is called single-valued if it contains a unique element for every game in the class.

We now introduce the simplest monotonicity requirement that we can ask of a solution. Let $\phi$ be a single-valued solution on a class of games $\Gamma_{0}$. We say that solution $\phi$ satisfies the aggregate monotonicity property (Meggido, 1974) if the following holds: for all $v, w \in \Gamma_{0}$, such that $v(S)=w(S)$ for all $S \neq N$ and $v(N)<w(N)$, then $\phi_{i}(v) \leq \phi_{i}(w)$ for all $i \in N$.

Given a vector $x \in \mathbb{R}^{N}$, the excess of a coalition $S$ at $x$ in a game $v$ is defined as $e(S, x):=v(S)-x(S)$. Let $\theta(x)$ be the vector of all excesses at $x$ arranged in non-increasing order of magnitude. The lexicographic order $\prec_{L}$ between two vectors $x$ and $y$ is defined by $x \prec_{L} y$ if there exists an index $k$ such that $x_{l}=y_{l}$ for all $l<k$ and $x_{k}<y_{k}$ and the weak lexicographic order $\preceq_{L}$ by $x \preceq_{L} y$ if $x \prec_{L} y$ or $x=y$.

Schmeidler (1969) introduced the nucleolus of a game $v$, denoted by $\nu(N, v)$, as the imputation that lexicographically minimizes the vector of non-increasingly ordered excesses over the set of imputations. In formula:

$$
\{\nu(N, v)\}=\left\{x \in I(N, v) \mid \theta(x) \preceq{ }_{L} \theta(y) \text { for all } y \in I(N, v)\right\} \text {. }
$$

For any game $v$ with a nonempty imputation set, the nucleolus is a singlevalued solution, is contained in the kernel and lies in the core provided that the core is nonempty. In the class of veto balanced games the kernel, the prekernel and the nucleolus coincide (see Arin and Feltkamp (1997)).

\subsection{A non-cooperative game (Arin and Feltkamp, 2007)}

Given a veto balanced game $(N, v)$ and an order of players, we will define a tree game associated to the TU game and denote it by $G(N, v)$. The game has $n$ stages and in each stage only one player takes an action. In the first stage, a veto player announces a proposal $x^{1}$ that belongs to the set of feasible and nonnegative allocations of the game $(N, v)$. In the next stages the responders accept or reject sequentially. If a player, say $i$, accepts the proposal $x^{t-1}$ at stage $t$, he leaves the game with the payoff $x_{i}^{t-1}$ and for the next stage 
the proposal $x^{t}$ coincides with the proposal at $t-1$, that is $x^{t-1}$. If player $i$ rejects the proposal then a two-person TU game is formed with the proposer and player $i$. In this two-person game the value of the grand coalition is $x_{1}^{t-1}+x_{i}^{t-1}$ and the value of the singletons is obtained by applying the DavisMaschler reduced game ${ }^{1}$ (Davis and Maschler (1965)) given the game $(N, v)$ and the allocation $x^{t-1}$. Player $i$ will receive as payoff the restricted standard solution of this two-person game. Once all the responders have played and consequently have received their payoffs the payoff of the proposer is also determined.

Formally, the resulting outcome of playing the game can be described by the following algorithm.

Input : a veto balanced game $(N, v)$ with a veto player, player 1 , and an order on the set of remaining players (responders).

Output : a feasible and nonnegative allocation $x$.

1. Start with stage 1 . Player 1 makes a feasible and nonnegative proposal $x^{1}$ (not necessarily an imputation). The superscript denotes at which stage the allocation emerges as the proposal in force.

2. In the next stage the first responder (say, player 2) says yes or no to the proposal. If he says yes he receives the payoff $x_{2}^{1}$, leaves the game, and $x^{2}=x^{1}$.

\footnotetext{
${ }^{1}$ Let $(N, v)$ be a game, $T$ a subset of $N$ such that $T \neq N, \emptyset$, and $x$ a feasible allocation.
} Then the Davis-Maschler reduced game with respect to $N \backslash T$ and $x$ is the game $(N \backslash$ $T, v_{x}^{N \backslash T}$ ) where

$$
v_{x}^{N \backslash T}(S):=\left\{\begin{array}{l}
0 \quad \text { if } S=\emptyset \\
v(N)-x(T) \text { if } S=N \backslash T \\
\max _{Q \subseteq T}\{v(S \cup Q)-x(Q)\} \quad \text { for all other } S \subset N \backslash T .
\end{array}\right.
$$

Note that we have defined a modified Davis-Maschler reduced game where the value of the grand coalition of the reduced game is $x(N \backslash T)$ instead of $v(N)-x(T)$. If $x$ is efficient both reduced games coincide. See also Peleg (1986). 
If he says no he receives the payoff ${ }^{2}$

$$
\begin{array}{r}
y_{2}=\max \left\{0, \frac{1}{2}\left[x_{1}^{1}+x_{2}^{1}-v_{x^{1}}(\{1\})\right]\right\} \text { where } \\
v_{x^{1}}(\{1\})=\max _{1 \in S \subseteq N \backslash\{2\}}\left\{v(S)-x^{1}(S \backslash\{1\})\right\} \\
\text { Now, } x_{i}^{2}=\left\{\begin{array}{cc}
x_{1}^{1}+x_{2}^{1}-y_{2} & \text { for player } 1 \\
y_{2} & \text { for player } 2 \\
x_{i}^{1} & \text { if } i \neq 1,2 .
\end{array}\right.
\end{array}
$$

3. Let the stage $t$ where responder $k$ plays, given the allocation $x^{t-1}$. If he says yes he receives the payoff $x_{k}^{t-1}$, leaves the game, and $x^{t}=x^{t-1}$. If he says no he receives the payoff

$$
\begin{array}{r}
y_{k}=\max \left\{0, \frac{1}{2}\left[x_{1}^{t-1}+x_{k}^{t-1}-v_{x^{t-1}}(\{1\})\right]\right\} \text { where } \\
v_{x^{t-1}}(\{1\})=\max _{1 \in S \subseteq N \backslash\{k\}}\left\{v(S)-x^{t-1}(S \backslash\{1\})\right\} . \\
\text { Now, } x_{i}^{t}=\left\{\begin{array}{cc}
x_{1}^{t-1}+x_{k}^{t-1}-y_{k} & \text { for player } 1 \\
y_{k} & \text { for player } k . \\
x_{i}^{t-1} & \text { if } i \neq 1, k
\end{array}\right.
\end{array}
$$

4. The game ends when stage $n$ is played and we define $x^{n}(N, v)$ as the vector with coordinates $\left(x_{j}^{n}\right)_{j \in N}$.

In this game we assume that the conflict between the proposer and a responder is solved bilaterally. In the event of conflict, the players face a two-person TU game that shows the strength of each player given that the rest of the responders are passive. Once the game is formed the allocation proposed for the game is a normative proposal, a kind of restricted standard solution $^{3}$.

\footnotetext{
${ }^{2}$ Note that, since 1 is a veto player, $v_{x^{t}}(\{i\})=0$ for any proposal $x^{t}$ and any player $i \neq 1$.

${ }^{3}$ In some sense the game is a hybrid of non-cooperative and cooperative games, since the outcome in case of conflict is not obtained as the equilibrium of a non-cooperative game.
} 


\subsection{The Nash outcomes of the game}

Given a game $(N, v)$ and a feasible allocation $x$ we define $f_{i j}$ as follows:

$$
f_{i j}(x,(N, v))=\min _{i \in S \subseteq N \backslash\{j\}}\{x(S)-v(S)\} .
$$

If there is no confusion we write $f_{i j}(x)$ instead of $f_{i j}(x,(N, v))$. Note that, if $j$ is a veto player, $f_{i j}(x)=x_{i}$.

The concept $f_{i j}(x)$ is closely related to the surplus of $i$ against $j$ at $x$ (terminology of Maschler, 1992), $s_{i j}(x)=\max _{i \in S \subseteq N \backslash\{j\}}\{v(S)-x(S)\}$. Given a coalition $S, x(S)-v(S)$ is the satisfaction of coalition $S$ at $x$; thus $f_{i j}(x)$ is the minimum satisfaction of a coalition that contains $i$ but not $j$. The higher $f_{i j}(x)$, the better $i$ is treated by the allocation $x$ in comparison with $j$. The kernel can be defined as the set of imputations such that $f_{j i}(x)>f_{i j}(x)$ implies $x_{j}=v(\{j\})$.

The set of bilaterally balanced allocations for player $i$ is

$$
F_{i}(N, v)=\left\{x \in D(N, v): f_{j i}(x) \geq f_{i j}(x) \text { for all } j \neq i\right\}
$$

while the set of optimal allocations for player $i$ in the set $F_{i}(N, v)$ is defined as follows:

$$
B_{i}(N, v)=\underset{x \in F_{i}(N, v)}{\arg \max } x_{i} .
$$

In the class of veto-balanced games, $F_{i}(N, v)$ is a nonempty and compact set for all $i$, thus the set $B_{i}(N, v)$ is nonempty.

Theorem 1 (Arin and Feltkamp, 2007) Let $(N, v)$ be a veto balanced $T U$ game and let $G(N, v)$ be its associated tree game. Let $z$ be a feasible and nonnegative allocation. Then $z$ is a Nash (SPE) outcome if and only if $z \in B_{1}(N, v)$.

The idea behind this result is the following. As shown in Arin and Feltkamp (2007), the restricted standard solution that is applied if player $i$ rejects a proposal in round $t$ results in $f_{1 i}\left(x^{t}\right)=f_{i 1}\left(x^{t}\right)$, unless this would mean a negative payoff for player $i$, in which case $f_{i 1}\left(x^{t}\right)>f_{1 i}\left(x^{t}\right)$ and $x_{i}^{t}=0$. Hence, it is in player $i$ 's interest to reject any proposal with $f_{1 i}\left(x^{t-1}\right)>$ 
$f_{i 1}\left(x^{t-1}\right)$ and to accept all other proposals. Since player $i$ rejects proposals with $f_{1 i}\left(x^{t-1}\right)>f_{i 1}\left(x^{t-1}\right)$ and this rejection results in $f_{1 i}\left(x^{t}\right)=f_{i 1}\left(x^{t}\right)$, the proposal in force after $i$ has the move always satisfies $f_{i 1}\left(x^{t}\right) \geq f_{1 i}\left(x^{t}\right)$. Subsequent actions by players moving after $i$ can only reduce $f_{1 i}($.$) , hence$ $f_{i 1}\left(x^{n}\right) \geq f_{1 i}\left(x^{n}\right)$. Player 1 then maximizes his own payoff under the constraint that the final allocation has to be bilaterally balanced.

The elements of $B_{1}(N, v)$ are not necessarily efficient. Indeed, in some cases, the set $B_{1}(N, v)$ contains no efficient allocations. The existence of an efficient equilibrium is not guaranteed because the nucleolus does not satisfy aggregate monotonicity for the class of veto balanced games. If $(N, v)$ is such that decreasing the value of the grand coalition (keeping the values of other coalitions constant) leads to the nonincreasing of the nucleolus payoff for player 1, the nucleolus of the game is a Nash outcome (Arin and Feltkamp, 2007, theorem 13).

\section{A new game: sequential proposals}

\subsection{The model}

We extend the previous model to $n$ periods. Given a veto balanced game with a proposer and an order on the set of responders we will construct a tree game, denoted by $G^{n}(N, v)$. In this game the proposer can make $n$ sequential proposals, and each proposal is answered by the responders as in the previous model.

Formally, the resulting outcome of playing the game can be described by the following algorithm.

Input : a veto balanced game $(N, v)$ with a veto player, player 1 , as proposer, and an order on the set of the remaining players (responders) which may be different for different periods.

Output : a feasible and nonnegative allocation $x$.

1. Start with period 1 . Given a veto balanced TU game $(N, v)$ and the order on the set of responders corresponding to period 1, players play the game $G(N, v)$. The outcome of this period determines the veto 
balanced TU game for the second period, denoted by $\left(N, v^{2, x^{1}}\right)$, where $v^{2, x^{1}}(S)=\max \left\{0, \min \left\{v(N)-x^{1}(N), v(S)-x^{1}(S)\right\}\right\}$ and $x^{1}$ is the final outcome obtained in the first period. Note that by construction, the game $\left(N, v^{2, x^{1}}\right)$ is a veto balanced game where player 1 is a veto player. Then go to the next step. The superscripts in the characteristic function denote at which period and after which outcome the game is considered as the game in force. If no confusion arises we write $v^{2}$ instead of $v^{2, x^{1}}$.

2. Let the period be $t(t<n+1)$ and the TU game $\left(N, v^{t, x^{t-1}}\right)$. We play the game $G\left(N, v^{t, x^{t-1}}\right)$ and define the veto balanced TU game $\left(N, v^{t+1, x^{t}}\right)$ where $v^{t+1}(S)=\max \left\{0, \min \left\{v^{t}(N)-x^{t}(N), v^{t}(S)-x^{t}(S)\right\}\right\}$ and $x^{t}$ is the final outcome obtained in period $t$. Then go to the next step.

3. The game ends after stage $n$ of period $n$. (If at some period before $n$ the proposer makes an efficient proposal (efficient according to the TU game underlying at this period) the game is trivial for the rest of the periods).

4. The outcome is the sum of the outcomes generated at each period.

In this paper we focus on games with $n$ periods (the number of players). Games with a different number of periods can be easily defined and will be used in some of the proofs. We refer to the game with $m$ proposals as $G^{m}(N, v)$, where $m \geq 1$.

\subsection{A serial rule for veto balanced games}

We now introduce a solution concept defined on the class of veto balanced games and denoted by $\phi$. Somewhat surprisingly, this solution will be related to the non-cooperative game with sequential proposals.

Let $(N, v)$ be a veto balanced game where player 1 is a veto player. Define for each player $i$ a value $d_{i}$ as follows:

$$
d_{i}:=\max _{S \subseteq N \backslash\{i\}} v(S) .
$$


Because 1 is a veto player, $d_{1}=0$. Let $d_{n+1}:=v(N)$ and rename the remaining players according to the nondecreasing order of those values. That is, player 2 is the player with the lowest value and so on. The solution $\phi$ associates to each veto balanced game, $(N, v)$, the following payoff vector:

$$
\phi_{l}=\sum_{i=l}^{n} \frac{d_{i+1}-d_{i}}{i} \text { for all } l \in\{1, \ldots, n\} .
$$

The following example illustrates how the solution behaves.

Example 2 Let $N=\{1,2,3,4\}$ be a set of players and consider the following 4-person veto balanced game $(N, v)$ where

$$
v(S)=\left\{\begin{array}{cc}
8 & \text { if } S \in\{\{1,2,3\},\{1,2,4\}\} \\
6 & \text { if } S=\{1,3,4\} \\
12 & \text { if } S=N \\
0 & \text { otherwise. }
\end{array}\right.
$$

Computing the vector of $d$-values we get:

$$
\left(d_{1}, d_{2}, d_{3}, d_{4}, d_{5}\right)=(0,6,8,8,12) .
$$

Applying the formula,

$$
\begin{array}{lrrr}
\phi_{1}= & \frac{d_{2}-d_{1}}{1}+\frac{d_{3}-d_{2}}{2}+\frac{d_{4}-d_{3}}{3}+\frac{d_{5}-d_{4}}{4}=8 \\
\phi_{2}= & \frac{d_{3}-d_{2}}{2}+\frac{d_{4}-d_{3}}{3}+\frac{d_{5}-d_{4}}{4}=2 \\
\phi_{3}= & \frac{d_{4}-d_{3}}{3}+\frac{d_{5}-d_{4}}{4}=1 \\
\phi_{4}= & & \frac{d_{5}-d_{4}}{4}=1 .
\end{array}
$$

The formula suggests a serial rule principle (cf. Moulin and Shenker, 1992). Since it is not possible for any coalition to obtain a payoff above $d_{i}$ without player $i$ 's cooperation, we can view player $i$ as having a right over the amount $v(N)-d_{i}$. The value $v(N)$ is divided in segments $\left(d_{2}-d_{1}\right.$, $\left.d_{3}-d_{2}, \ldots, v(N)-d_{n}\right)$ and each payoff segment is divided equally among the players that have a right over it. 
In the class of veto balanced games, the solution $\phi$ satisfies some wellknown properties such as nonemptiness, efficiency, anonymity and equal treatment of equals among others. It also satisfies aggregate monotonicity. ${ }^{4}$

We now show that $\phi$ provides a core allocation for veto balanced games.

Lemma 3 (Arin and Feltkamp, 2012) Let $(N, v)$ be a veto balanced $T U$ game. Then $\phi(N, v) \in C(N, v)$.

Proof. We need to prove that for any coalition $S$ it holds that $v(S) \leq$ $\sum_{l \in S} \phi_{l}$. Note that $\sum_{l=1}^{k-1}\left(\phi_{l}-\phi_{k}\right)=d_{k}-d_{1}$; since 1 is a veto player we have $d_{1}=0$ and hence $\sum_{l=1}^{k-1}\left(\phi_{l}-\phi_{k}\right)=d_{k}$. Let $S$ be a coalition and let $d_{j}$ be such that $d_{j}=\min _{i \notin S} d_{i}$. Let $k$ be the first player for which $d_{k}=d_{j}$. Therefore, $\{1,2, \ldots, k-1\} \subseteq S$. By definition $v(S) \leq d_{k}=\sum_{l=1}^{k-1}\left(\phi_{l}-\phi_{k}\right) \leq \sum_{l=1}^{k-1} \phi_{l} \leq$ $\sum_{l \in S} \phi_{l} \cdot 5$

The next section shows that $\phi(N, v)$ is the unique equilibrium outcome assuming that all responders act as myopic maximizers and the proposer plays a best response taking this into account.

\subsection{Myopic Best Response Equilibrium}

The first approach we take to solve the non-cooperative game with sequential proposals consists of analyzing myopic behavior of the responders. Responders behave myopically when they act as payoff maximizers in each period without considering the effect of their actions on future periods.

Suppose all responders maximize payoffs myopically for each period and that the proposer plays optimally taking into account that the responders are myopic maximizers. We call such a strategy profile a myopic best response

\footnotetext{
${ }^{4}$ For a definition of those properties, see Peleg and Sudhölter (2003). It is not the aim of this paper to provide an axiomatic analysis of the solution. Arin and Feltkamp (2012) characterize the solution in the domain of veto balanced games by core selection and a monotonicity property.

${ }^{5}$ Because the game is balanced, $v(N) \geq d_{n}$, which implies $\phi_{k} \geq 0$ for all $k$.
} 
equilibrium (MBRE). We will show in this section that all MBRE lead to the same outcome.

If there is only one period in the game, myopic and rational behavior coincide. This means that the following lemma holds if responders behave myopically.

Lemma 4 (Arin and Feltkamp, 200\%, lemmas 2 and 3) Let $(N, v)$ be a veto balanced $T U$ game, and $G^{n}(N, v)$ its associated tree game. At any period $k$ and stage $t$, the responder (say, i) will accept $x_{t-1}^{k}$ if $f_{i 1}\left(x_{t-1}^{k} ; v^{k, x^{k-1}}\right)>$ $f_{1 i}\left(x_{t-1}^{k} ; v^{k, x^{k-1}}\right)$, and will reject it if $f_{i 1}\left(x_{t-1}^{k} ; v^{k, x^{k-1}}\right)<f_{1 i}\left(x_{t-1}^{k} ; v^{k, x^{k-1}}\right)$ in a MBRE. If $f_{i 1}\left(x_{t-1}^{k} ; v^{k, x^{k-1}}\right)=f_{1 i}\left(x_{t-1}^{k} ; v^{k, x^{k-1}}\right)$, the responder is indifferent between accepting and rejecting since both decisions lead to the same outcome. Also, the final outcome of any period $k$ is such that $f_{i 1}\left(x^{k} ; v^{k, x^{k-1}}\right) \geq$ $f_{1 i}\left(x^{k} ; v^{k, x^{k-1}}\right)$ for all $i$.

The notion of balanced proposals will play a central role in the proofs of the main results.

Definition 5 Let $(N, v)$ be a veto balanced TU game. Consider its associated tree game $G^{n}(N, v)$. Given a period $k$, a proposal $x$ is balanced if it results as the final outcome of period $k$ regardless of the actions of the responders.

Balanced proposals coincide with the nucleolus (kernel) of special games. In the class of veto-rich games (games with at least one veto player and a nonempty set of imputations) the kernel and the nucleolus coincide (Arin and Feltkamp, 1997). Therefore we can define the nucleolus as

$$
\nu(N, v)=\left\{x \in I(N, v): f_{i j}(x)<f_{j i}(x) \Longrightarrow x_{j}=0\right\} .
$$

We use this alternative definition of the nucleolus in the proof of the following lemma.

Lemma 6 Let $(N, v)$ be a veto balanced TU game. Consider the associated game $G^{n}(N, v)$. Given a period $k$, a proposal $x$ is balanced if and only if it coincides with the nucleolus of the game $(N, w)$, where $w(S)=v^{k}(S)$ for all $S \neq N$ and $w(N)=x(N)$. 
Proof. Assume that $x$ is a balanced proposal in period $k$ with the game $\left(N, v^{k}\right)$.

a) Let $l$ be a responder for which $x_{l}=0$. If whatever the response of player $l$ the proposal does not change then $f_{1 l}(x) \leq 0=x_{l}=f_{l 1}(x)$.

b) Let $m$ be a responder for which $x_{m}>0$. If whatever the response of player $m$ the proposal does not change then $f_{1 m}(x)=x_{m}=f_{m 1}(x)$.

Therefore, the bilateral kernel conditions are satisfied for the veto player. Lemma 12 in Arin and Feltkamp (2007) shows that if the bilateral kernel conditions are satisfied between the veto player and the rest of the players then the bilateral kernel conditions are satisfied between any pair of players.

Therefore, $x$ is the kernel (nucleolus) of the game $(N, w)$. The converse statement can be proven in the same way.

We now show that, by making balanced proposals, the proposer can secure the payoff provided by the serial rule $\phi$.

Lemma 7 Let $(N, v)$ be a veto balanced $T U$ game and $G^{n}(N, v)$ its associated tree game. Let $z$ be a Nash outcome of the game $G^{n}(N, v)$. Then $z_{1} \geq \phi_{1}=$ $\sum_{i=1}^{n} \frac{d_{i+1}-d_{i}}{i}$.

Proof. The result is based on the fact that the proposer has a strategy with which he secures the payoff $\sum_{i=1}^{n} \frac{d_{i+1}-d_{i}}{i}$ regardless of the strategies of the rest of the players by making a sequence of balanced proposals.

The strategy is the following. At each period $t,(t \in\{1, \ldots, n\})$ consider the set $S_{t}=\{l: l \leq t\}$ and the proposal $x^{t}$, defined as follows:

$$
x_{l}^{t}=\left\{\begin{array}{cc}
\frac{d_{t+1}-d_{t}}{t} & \text { for all } l \in S_{t} \\
0 & \text { otherwise }
\end{array}\right.
$$

whenever $x^{t}$ is feasible and propose the 0 vector otherwise.

It can be checked immediately that in each stage the proposed allocation will be the final allocation independently of the answers of the responders and independently of the order of those answers. The proposals are balanced proposals. 
For example, in period 1 , the proposal is $\left(d_{2}, 0, \ldots, 0\right)$. Because 1 is a veto player, $f_{i 1}()=$.0 for all $i$. As for $f_{1 i}($.$) , because all players other than 1$ are getting 0 , the coalition of minimum satisfaction of 1 against $i$ is also the coalition of maximum $v(S)$ with $i \notin S$. Call this coalition $S^{*}$. By definition, $v\left(S^{*}\right)=d_{i} \geq d_{2}$ and $f_{1 i}()=.x\left(S^{*}\right)-v\left(S^{*}\right)=d_{2}-d_{i} \leq 0$. Thus, $f_{i 1}(.) \geq f_{1 i}($. for all $i$ and the outcome of period 1 is $\left(d_{2}, 0, \ldots, 0\right)$ regardless of responders' behavior.

In period 2 we have a game $v^{2}$ with the property that $v^{2}(S)>0$ implies $v^{2}(S)=v^{1}(S)-d_{2}$ for all $S$. Thus, player 2 is a veto player in $v^{2}$. Player 1 proposes $\left(\frac{d_{3}-d_{2}}{2}, \frac{d_{3}-d_{2}}{2}, 0, \ldots, 0\right)$. If player 2 rejects, we have $f_{12}()=.\frac{d_{3}-d_{2}}{2}-$ $0=f_{21}($.$) . As for other players i \neq 1,2$, when computing $f_{1 i}$ we take into account that any coalition of positive value must include 1 and 2 . Since players other than 1 and 2 are getting 0 , the coalition 1 uses against $i$ is $S^{*} \in \arg \max _{S: i \notin S} v(S)$. By definition, $v\left(S^{*}\right)=d_{i}$ and $v^{2}\left(S^{*}\right)=d_{i}-d_{2}$. Then $f_{1 i}()=.x\left(S^{*}\right)-v^{2}\left(S^{*}\right)=\left(d_{3}-d_{2}\right)-\left(d_{i}-d_{2}\right)=d_{3}-d_{i} \leq 0$.

In period 3, player 3 has become a veto player and the same process can be iterated until period $n$.

Therefore, this strategy of the proposer determines the total payoff of all the players, that is, the final outcome of the game $G^{n}(N, v)$. This final outcome coincides with the solution $\phi$.

This proof, together with lemma 6 , suggests a new interpretation of the serial rule. At each stage the proposal coincides with the nucleolus of a veto-rich game. Formally,

$$
\phi(N, v)=\sum_{i=1}^{n} \nu\left(N, w^{i}\right)
$$

where the games $\left(N, w^{i}\right)$ are defined as follows: $w^{1}(N)=d_{2}$ and $w^{1}(S)=$ $v(S)$ otherwise. For $i: 2, . ., n$ :

$$
w^{i}(S)=\left\{\begin{array}{cc}
d_{i+1}-d_{i} & \text { if } S=N \\
\max \left\{0, w^{i-1}(S)-\sum_{l \in S} \nu_{l}\left(N, w^{i-1}\right)\right\} & \text { otherwise. }
\end{array}\right.
$$

Remark 8 The serial rule can also be obtained by making balanced proposals if the game has $n-1$ periods. 
This is because the proposer can combine the first two proposals in the proof of lemma 7 by proposing $\left(d_{2}+\frac{d_{3}-d_{2}}{2}, \frac{d_{3}-d_{2}}{2}, 0, \ldots, 0\right)$ in the first period.

We now show that the proposer cannot improve upon $\phi$ in a MBRE.

Proposition 9 Let $(N, v)$ be a veto balanced $T U$ game and $G^{n}(N, v)$ its associated tree game. Let $z$ be a MBRE outcome of the game $G^{n}(N, v)$. Then $z_{1} \leq \phi_{1}=\sum_{i=1}^{n} \frac{d_{i+1}-d_{i}}{i}$

We will prove this proposition by a series of lemmas. We will first show that any payoff player 1 can obtain given myopic responder behavior can also be obtained by making balanced proposals: player 1 can cut the payoff of other players until a balanced proposal is obtained at no cost to himself (lemma 10). We will then show that the payoff player 1 can get with balanced proposals in a given period cannot exceed the serial rule of a particular TU game $w^{t}$. Finally, we will check that the sum of the serial rules of the games $w^{t}$ cannot exceed the serial rule of the original game $(N, v)$ (lemma 16), hence player 1's total payoff cannot exceed $\phi_{1}(N, v)$.

Lemma 10 Let $(N, v)$ be a veto balanced game. Consider the associated game with $m$ stages $G^{m}(N, v)$. Let $z=\sum_{1}^{m} x^{t}$ be an outcome resulting from some strategy profile. Assume that the final outcome of any period $t, x^{t}$, is such that for any player $l, x_{l}^{t} \geq f_{1 l}\left(x^{t}, v^{t}\right)$. Then there exists $y$ such that $y_{1}=z_{1}, y=\sum_{1}^{m} q^{t}$ where $q^{t}$ is a balanced proposal for period $t$.

Proof. If $\left(x^{1}, x^{2}, \ldots, x^{m}\right)$ is a sequence of balanced proposals the proof is done.

Suppose that $\left(x^{1}, x^{2}, \ldots, x^{m}\right)$ is not a sequence of balanced proposals. This means that for some $x^{t}$ and for some $i \neq 1$ it holds that $x_{i}^{t}>f_{1 i}\left(x^{t}, v^{t}\right)$ and $x_{i}^{t}>0$. Let $k$ be the first period where such result holds. Therefore, $\left(x^{1}, x^{2}, \ldots, x^{k-1}\right)$ is a sequence of balanced proposals. We will construct a balanced proposal where the payoff of the proposer does not change.

Since $f_{i 1}\left(x^{k}\right)=x_{i}^{k}$, by reducing the payoff of player $i$ we can construct a new allocation $y^{k}$ such that $f_{1 i}\left(y^{k}\right)=f_{i 1}\left(y^{k}\right)$ or $f_{1 i}\left(y^{k}\right)<f_{i 1}\left(y^{k}\right)$ and $y_{i}^{k}=0$. In any case, $x_{1}^{k}=y_{1}^{k}$ and the payoff of the proposer does not change. Note 
also that reducing $i$ 's payoff can only lower $f_{1 j}\left(y^{k}\right)$, so it is still the case that $f_{1 j}\left(y^{k}\right) \leq f_{j 1}\left(y^{k}\right)$ for all $j$.

Now, if there exists another player $l$ such that $f_{1 l}\left(y^{k}\right)<f_{l 1}\left(y^{k}\right)$ and $y_{l}^{k}>0$ we construct a new allocation $z^{k}$ such that $f_{1 l}\left(z^{k}\right)=f_{l 1}\left(z^{k}\right)$ or $f_{1 i}\left(z^{k}\right)<f_{i 1}\left(z^{k}\right)$ and $z_{i}^{k}=0$. Note that $z_{1}^{k}=y_{1}^{k}$. Repeating this procedure we will end up with a balanced allocation. If $q^{k}$ is the final outcome of this procedure, $q^{k}$ is the nucleolus of the game $\left(N, w^{k}\right)$ where $w^{k}(N)=q^{k}(N)$ and $w^{k}(S)=v^{k}(S)$ for all $S \neq N$.

The TU game $\left(N, w^{k+1}\right)$ resulting after proposing $q^{k}$ satisfies that $w^{k+1}(S) \geq$ $v^{k+1}(S)$ for all $S \ni 1$. Therefore, $f_{1 i}\left(x, w^{k+1}\right) \leq f_{1 i}\left(x, v^{k+1}\right)$ for any feasible allocation $x$, and $x_{l}^{k+1} \geq f_{1 l}\left(x^{k+1}, w^{k+1}\right)$ for all $l$.

Consider the game $\left(N, w^{k+1}\right)$ and the payoff $x^{k+1}$. Suppose that $x_{i}^{k+1}>$ $f_{1 i}\left(x^{k+1}\right)$ for some $i \neq 1$ and $x_{i}^{k+1}>0$. We can repeat the same procedure of period $k$ until we obtain a balanced allocation $q^{k+1}$. The procedure can be repeated until the last period of the game to obtain the sequence of balanced proposals $\left(x^{1}, x^{2}, \ldots, x^{k-1}, q^{k}, \ldots, q^{m}\right)$.

Some interesting properties of balanced proposals:

Lemma 11 If $x^{t}$ is a balanced proposal, any player $i$ with $x_{i}^{t}>0$ will be a veto player at $t+1$.

This is because if $x^{t}$ is balanced we have $f_{1 i}\left(x^{t}, v^{t}\right)=x_{i}^{t}$, so that all coalitions that have a positive $v^{t}$ but do not involve $i$ have $v^{t}(S)<x^{t}(S)$. Thus, after the payoffs $x^{t}$ are distributed any coalition with positive value must involve $i$. Note that this result requires $x^{t}$ to be a balanced proposal and not merely the outcome of a MBRE. In a MBRE it may be the case that $f_{1 i}\left(x^{t}, v^{t}\right)<x_{i}^{t}$, and we cannot conclude anything about the sign of $f_{1 i}\left(x^{t}, v^{t}\right)$.

The next lemma establishes a relationship between balanced proposals in $G^{m}(N, v)$ and the serial rule. Suppose $x^{t}$ is a balanced proposal in period $t$. Consider the game $w^{t}$, where $w^{t}(S)=\min \left\{v^{t}(S), x^{t}(N)\right\}$. The serial rule of $w^{t}$ and the balanced proposal $x^{t}$ do not coincide in general. However, the set of players who receive a positive payoff in $x^{t}$ coincides with the set of players who receive a positive payoff according to the serial rule of $w^{t} .{ }^{6}$

\footnotetext{
${ }^{6}$ For example, consider the game with $N=\{1,2,3,4\}, v(1,2)=v(1,3)=2, v(1,2,3)=$
} 
Lemma 12 Let $(N, v)$ be a veto balanced TU game. Consider the associated game $G^{m}(N, v)$. Let $z=\sum_{1}^{m} x^{t}$ be the outcome resulting from some strategy profile with balanced proposals. Consider period $t$, its outcome $x^{t}$ and the game $\left(N, w^{t}\right)$ where $w^{t}(S)=\min \left\{v^{t}(S), x^{t}(N)\right\}$. Then it holds that $x_{k}^{t}>0$ if and only if $\phi_{k}\left(N, w^{t}\right)>0$.

Proof. a) If $x_{k}^{t}>0$ we need to prove that $d_{k}\left(N, w^{t}\right)<x^{t}(N)$, so that the serial rule of $w^{t}$ assigns a positive payoff to $k$.

Let $S \in \arg \max _{T \subseteq N \backslash\{k\}} v^{t}(T)$. Since $x^{t}$ is balanced we have $f_{1 k}\left(x^{t}\right)=$ $x_{k}^{t}>0$ and that implies $x^{t}(S)>v^{t}(S)$ (otherwise $S$ could have been used to complain against $k$ ). Hence, $x^{t}(N) \geq x^{t}(S)>v^{t}(S)=d_{k}\left(v^{t}\right)=d_{k}\left(w^{t}\right)$, where the last equality follows from lemma $11 .^{7}$

b) If $x_{k}^{t}=0$ we need to prove that $d_{k}\left(N, w^{t}\right)=x^{t}(N)$. Since $x^{t}$ is balanced, $f_{1 k}\left(x^{t}, v^{t}\right) \leq 0$. Let $P$ be a coalition associated to $f_{1 k}\left(x^{t}, v^{t}\right)$. Because $f_{1 k}\left(x^{t}, v^{t}\right) \leq 0, x^{t}(P) \leq v^{t}(P)$. Coalition $P$ must contain all players receiving a positive payoff at $x^{t}$ (otherwise $x^{t}$ is not balanced since $P$ can be used against any player outside $P)$. Therefore $x^{t}(N)=x^{t}(P) \leq v^{t}(P)$. Because of the way $w^{t}$ is defined it cannot exceed $x^{t}(N)$, so $x^{t}(N)=w^{t}(P)=$ $d_{k}\left(w^{t}\right)$ and $k$ receives 0 according to the serial rule of $w^{t}$.

The following lemma concerns a property of the serial rule. By definition, the serial rule is such that $d_{k}$ is divided among players $\{j \in N, j<k\}$. Above $d_{k}$, player $k$ and any player $j<k$ get the same payoff.

Lemma 13 For any player $k$ we have $\sum_{i \in\{1,2, \ldots, k-1\}} \phi_{i}=d_{k}+(k-1) \phi_{k}$. Hence, $\sum_{i \in\{1,2, \ldots, k-1\}} \phi_{i} \geq d_{k}+\phi_{k}$. The latter inequality is strict except if $k=2$ or $\phi_{k}=0$.

$6, v(1,2,3,4)=10$ and $v(S)=0$ otherwise. The proposal $x=(2,1.5,1.5,0)$ is a balanced proposal with a total payoff distributed of 5 (and, because of lemma 6 and the uniqueness of the nucleolus, it is the only balanced proposal that distributes a total payoff of 5 ). The game $w$ associated to this proposal is identical to $v$ except that $w(1,2,3)=w(N)=5$. Its serial rule is $(3,1,1,0)$, which is different from the balanced proposal but gives a positive payoff to the same set of players.

${ }^{7}$ Because $x^{t}$ is a balanced proposal, the $d$-values of $w^{t}$ coincide with the $d$-values of $v^{t}$ for all players receiving a positive payoff. Any player $j$ that is receiving a positive payoff at $t$ will be veto at $t+1$ (lemma 11). The values $d_{j}\left(w^{t}\right)$ and $d_{j}\left(v^{t}\right)$ can only differ if $v^{t}(S)>x^{t}(N)$ for some $S$ such that $j \notin S$, but then player $j$ would not be veto at $t+1$. 
The next lemma tell us that, given a strategy profile with balanced proposals, the proposer cannot get more than the serial rule of the games $w^{t}$.

Lemma 14 Let $(N, v)$ be a veto balanced TU game. Consider the associated game with $m$ stages $G^{m}(N, v)$. Let $z=\sum_{1}^{m} x^{t}$ be an outcome resulting from balanced proposals. Consider period $t$, its outcome $x^{t}$ and the game $\left(N, w^{t}\right)$ where $w^{t}(S)=\min \left\{v^{t}(S), x^{t}(N)\right\}$. Then $x_{1}^{t} \geq \phi_{1}\left(N, w^{t}\right)$ implies $x_{l}^{t} \geq \phi_{l}\left(N, w^{t}\right)$ for all $l \in N$.

Proof. Let $T$ be the set of veto players in $\left(N, w^{t}\right)$, and let $M=\left\{l_{1}, \ldots, l_{m}\right\}$ be the ordered (according to the $d$-values of $\left(N, w^{t}\right)$ ) set of nonveto players that have received a positive payoff at $x^{t}$. That is, $d_{l_{1}} \leq \ldots \leq d_{l m}{ }^{8}$

Suppose $x_{1}^{t} \geq \phi_{1}\left(N, w^{t}\right)$. Since $x^{t}$ is balanced, $x_{1}^{t}=x_{i}^{t}$ for all $i \in T$, thus if $x_{1}^{t} \geq \phi_{1}\left(N, w^{t}\right)$ it follows that $x_{i}^{t} \geq \phi_{i}\left(N, w^{t}\right)$ for all $i \in T$.

We now want to prove that $x_{i}^{t} \geq \phi_{i}\left(N, w^{t}\right)$ for all $i \in M$. We will do it by induction.

Consider the responder $l_{1}$. Since $x^{t}$ is balanced we have $f_{1 l_{1}}\left(x^{t}\right)=x_{l_{1}}^{t}$. If the coalition associated to $f_{1 l_{1}}$ has a value of 0 , it follows that $x_{1}^{t}=x_{l_{1}}^{t}$ so $x_{l_{1}}^{t} \geq \phi_{l_{1}}\left(N, w^{t}\right)$. If the coalition 1 is using has a positive value, all veto players must be in it, so its payoff must be at least $|T| \phi_{1}\left(N, w^{t}\right)$, and its value (by definition of $\left.d_{l_{1}}\right)$ cannot exceed $d_{l_{1}}$. Hence, $f_{1 l_{1}}\left(x^{t}\right) \geq|T| \phi_{1}\left(N, w^{t}\right)-d_{l_{1}}$. Because of lemma 13, $|T| \phi_{1}\left(N, w^{t}\right)-d_{l_{1}} \geq \phi_{l_{1}}\left(N, w^{t}\right)$.

Now suppose the result $x_{i}^{t} \geq \phi_{i}\left(N, w^{t}\right)$ is true for all $i \in\left\{l_{1}, \ldots, l_{k-1}\right\}$. We will prove that $x_{l_{k}}^{t} \geq \phi_{l_{k}}\left(N, w^{t}\right)$. Let $S$ be a coalition such that $f_{1 l_{k}}\left(x^{t}\right)=$ $x^{t}(S)-v^{t}(S)$. As before, the result follows immediately if $v^{t}(S)=0$. If $v^{t}(S)>0$ it must be the case that $T \subseteq S$, but $S$ need not involve all players in $\left\{l_{1}, \ldots, l_{k-1}\right\}$. Denote $\left\{l_{1}, \ldots, l_{k-1}\right\}$ by $Q$. We consider two cases, depending on whether $Q \subseteq S$.

If $Q \subseteq S$, we have $x_{l_{k}}^{t}=f_{1 l_{k}}\left(x^{t}\right)=x^{t}(S)-v^{t}(S) \geq \sum_{i \in T \cup Q} \phi_{i}\left(N, w^{t}\right)-d_{l_{k}}$, where the last inequality uses the induction hypothesis. The set $T \cup Q$ contains all players with $d_{i}<d_{l_{k}}$. Hence, by lemma $13, \sum_{i \in T \cup Q} \phi_{i}\left(N, w^{t}\right)-$ $d_{l_{k}} \geq \phi_{l_{k}}\left(N, w^{t}\right)$.

\footnotetext{
${ }^{8}$ Recall that, because $x^{t}$ is a balanced proposal, the $d$-values of $w^{t}$ coincide with the $d$-values of $v^{t}$ for all players in $M$.
} 
If $Q \nsubseteq S$, there is a player $l_{p}<l_{k}$ such that $l_{p} \notin S$. Because $x^{t}$ is a balanced proposal, $x_{l_{p}}^{t}=f_{1 l_{p}}\left(x^{t}\right)$. Because the veto player can use $S$ to complain against $l_{p}, f_{1 l_{p}}\left(x^{t}\right) \leq f_{1 l_{k}}\left(x^{t}\right)=x_{l_{k}}$, hence $x_{l_{p}} \leq x_{l_{k}}$. By the induction hypothesis, $x_{l_{p}} \geq \phi_{l_{p}}\left(N, w^{t}\right)$. Since $d_{l_{p}} \leq d_{l_{k}}$ we also know that $\phi_{l_{p}}\left(N, w^{t}\right) \geq \phi_{l_{k}}\left(N, w^{t}\right)$, so that

$$
x_{l_{k}}=f_{1 l_{k}}\left(x^{t}\right) \geq f_{1 l_{p}}\left(x^{t}\right)=x_{l_{p}} \geq \phi_{l_{p}}\left(N, w^{t}\right) \geq \phi_{l_{k}}\left(N, w^{t}\right) .
$$

So far we have discussed the set of veto players and the set of nonveto players that are getting a positive payoff in $x^{t}$. For players in $N \backslash(T \cup M)$, we have shown in lemma 12 that $x_{j}^{t}=0$ implies $\phi_{j}\left(N, w^{t}\right)=0$, hence $x_{j}^{t} \geq$ $\phi_{j}\left(N, w^{t}\right)$ for all players.

Corollary 15 Putting lemma 14 together with the efficiency of the serial rule $\sum_{i \in N} \phi_{i}\left(N, w^{t}\right)=w^{t}(N)$, we see that $x_{1}^{t} \geq \phi_{1}\left(N, w^{t}\right)$ implies $x_{l}^{t}=\phi_{l}\left(N, w^{t}\right)$ for all $l \in N$. The only way in which player 1 can obtain the serial rule of $\left(N, w^{t}\right)$ with balanced proposals is that all players in the game obtain their serial rule payoff.

The sum of the serial rules of the games $w^{t}$ cannot exceed the serial rule of the original game. This is due to the following property of the serial rule:

Lemma 16 Consider the veto balanced $T U$ game $(N, v)$ and a finite set of positive numbers $\left(a_{1}, \ldots, a_{k}\right)$ such that $\sum_{l=1}^{k} a_{l}=v(N)$. Consider the following TU games: $\left(N, w^{1}\right),\left(N, w^{2}\right), \ldots,\left(N, w^{k}\right)$, where

$$
\begin{aligned}
& w^{1}(S)=\left\{\begin{array}{cc}
a_{1} & \text { if } S=N \\
\min \left\{a_{1}, v(S)\right\} & \text { otherwise }
\end{array}\right. \\
& w^{2}(S)=\left\{\begin{array}{cc}
a_{2} & \text { if } S=N \\
\min \left\{a_{2}, \max \left[0, v(S)-\sum_{i \in S} \phi_{i}\left(N, w^{1}\right)\right]\right\} & \text { otherwise }
\end{array}\right. \\
& w^{l}(S)=\left\{\begin{array}{cc}
a_{l} & \text { if } S=N \\
\min \left\{a_{l}, \max \left[0, v(S)-\sum_{m=1}^{l-1} \sum_{i \in S} \phi_{i}\left(N, w^{m}\right)\right]\right\} & \text { otherwise }
\end{array}\right.
\end{aligned}
$$

Then $\phi(N, v)=\sum_{i=1}^{k} \phi\left(N, w^{i}\right)$. 
In the lemma, we take $v(N)$ and divide it in $k$ positive parts, where $k$ is a finite number. Then we compute the serial rule for each of the $k$ games, and see that each player gets the same in total as in the serial rule of the original game.

The $k$ games are formed as follows: $w^{k}(N)$ is always $a_{k}$; the other coalitions have $v(S)$ minus what has been distributed so far according to the serial rule of the previous games, unless this would be negative (in which case the value is 0 ) or above $w^{k}(N)$ (in which case the value is $a_{k}$ ).

The idea of the proof is that player $i$ cannot get anything until $d_{i}$ has been distributed, and from that point on $i$ becomes veto. This happens regardless of the way $v(N)$ is divided into $k$ parts. For the same reason, if $\sum_{i=1}^{k} a_{l}<v(N)$, player 1 will get less than $\phi_{1}(N, v)$.

Note that lemma 16 refers to a sequence of TU games such that each game is obtained after distributing the serial rule payoffs for the previous game; the games $w^{t}$ in lemma 14 are obtained by subtracting balanced proposals from $w^{t-1}$. It turns out that the TU games involved are identical in both cases: the sequence $w^{t}$ depends only on the total amounts distributed $x^{1}(N), \ldots, x^{n}(N)$ (denoted by $a_{1}, \ldots, a_{n}$ in lemma 16). This is because the set of players that get a positive payoff at period $t$ is the same in both cases (lemma 12) and all these players become veto at period $t+1$ (lemma 11). Hence, any coalition with positive value at $t$ has $w^{t}(S)=\min \left(w^{t-1}(S)-x^{t-1}(N), x^{t}(N)\right)$ in both cases.

Putting the above lemmas together we can prove proposition 9. First, any payoff player 1 can achieve in a MBRE can be achieved by balanced proposals (lemma 10). Second, given that proposals are balanced, the payoff player 1 can get cannot exceed the sum of the serial rules of the games $w^{t}$ (lemma 14). Finally, the sum of the serial rules of the games $w^{t}$ cannot exceed the serial rule of the original game (lemma 16).

Given that player 1 can never get more than $\phi_{1}(N, v)$ in a MBRE by proposition 9 (and only if all players get their serial rule payoff by corollary $15)$, and that $\phi(N, v)$ is achievable by the sequential proposals described in lemma 7 , we have the following result. 
Theorem 17 Let $(N, v)$ be a veto balanced $T U$ game and $G^{n}(N, v)$ a tree game. Let $z$ be an outcome resulting from a MBRE of the game $G^{n}(N, v)$. Then $z=\phi(N, v)$.

Corollary 18 Let $(N, v)$ be a veto balanced TU game. Then $\phi_{1}(N, v) \geq$ $\nu_{1}(N, v)$.

Proof. In a MBRE, $\phi_{1}(N, v)$ coincides with the equilibrium payoff for the proposer in the game $G^{n}(N, v)$. This equilibrium payoff is at least as large as his equilibrium payoff in the game $G^{1}(N, v)$, because the proposer can always wait until period $n$ to divide the payoff. This equilibrium payoff is in turn at least as high as $\nu_{1}(N, v)$, because $\nu(N, v)$ is a balanced proposal.

\subsection{An example}

The next example illustrates that a MBRE need not be a subgame perfect equilibrium.

Example 19 Let $N=\{1,2,3,4,5\}$ a set of players and consider the following 5-person veto balanced game $(N, v)$ where

$$
v(S)=\left\{\begin{array}{cc}
36 & \text { if } S \in\{\{1,2,3,5\},\{1,2,3,4\}\} \\
31 & \text { if } S=\{1,2,4,5\} \\
51 & \text { if } S=N \\
0 & \text { otherwise. }
\end{array}\right.
$$

Computing the outcome associated to any MBRE we see that the proposer receives as payoff the amount $\phi_{1}(N, v)=121 / 6$. As we know, this result is true for any order of the responders. Suppose the order of responders is $2,3,4,5$. The following result holds given this order: If the responders play the game optimally (not necessarily as myopic maximizers) the proposer can get a higher payoff than the one provided by the MBRE outcome. Therefore, MBRE and SPE outcomes do not necessarily coincide.

The strategy is the following: The proposer offers nothing in the first three stages. In the 4 th period the proposal is: $(10,10,5,0,0)$. 
The responses of players 2, 4 and 5 do not change the proposal (even if the proposal faced by player 4 and 5 is a new one resulting from a rejection of player 3 ). If player 3 accepts this proposal, the TU game for the last period will be:

$$
w(S)=\left\{\begin{array}{cc}
11 & \text { if } S \in\{\{1,2,3,5\},\{1,2,3,4\}\} \\
11 & \text { if } S=\{1,2,4,5\} \\
26 & \text { if } S=N \\
0 & \text { otherwise. }
\end{array}\right.
$$

In the last period, myopic and rational behavior coincide, so the outcome must be an element of $B_{1}(N, w)$. It can be checked that $B_{1}(N, w)=$ $\{(5.5,5.5,0,0,0)\}$. Therefore, after accepting the proposal in period 4 , player 3 gets a total payoff of 5 .

If player 3 rejects the proposal, the outcome of the 4 th period is $(15,10,0,0,0)$ and the TU game for the last period is:

$$
u(S)=\left\{\begin{array}{cc}
11 & \text { if } S \in\{\{1,2,3,5\},\{1,2,3,4\}\} \\
6 & \text { if } S=\{1,2,4,5\} \\
26 & \text { if } S=N \\
0 & \text { otherwise }
\end{array}\right.
$$

As before, in the last period myopic and rational behavior coincide and the outcome must be an element of $B_{1}(N, u)$. It can be checked that $B_{1}(N, u)=$ $\{(5.2,5.2,5.2,5.2,5.2)\}$. Therefore, after rejecting the proposal player 3 gets a total payoff of 5.2 .

Therefore, rational behavior of player 3 implies a rejection of the proposal in the 4th period. This rejection is not a myopic maximizer's behavior. After the rejection of player 3 the proposer gets a payoff of 20.2 , higher than $121 / 6$. Hence, the outcome associated to MBRE is not the outcome of a SPE.

In the example above, the proposer finds a credible way to collude with player 3 in order to get a higher payoff than the one obtained by player 2 (a veto player). Player 2 cannot avoid this collusion since he is responding before player 3 . If he responded after player 3 , collusion between players 1 and 3 would no longer be profitable. This observation turns out to be crucial as we will see in the next section. 
Finally, consider the following profile of strategies: the proposer plays the strategy presented in Lemma 7 and the responders behave as myopic maximizers. This profile is a Nash equilibrium and its outcome is $\phi(N, v)$. Therefore:

Remark 20 The MBRE outcome is a Nash outcome.

\subsection{Myopic behavior and Subgame Perfect Equilibria}

The previous example shows that, in general, perfectly rational behavior and myopically rational behavior do not coincide. However, they do coincide when the model incorporates a requirement on the order of the responders. From now on, we assume that at each stage $t$ the order of the responders is given by the nonincreasing order of the $d$-values of the game $v^{t}$. That is, the order of the responders is not completely fixed in advance and can be different for different periods. Given this order, any veto responder can secure a payoff equal to or higher than the payoff obtained by the proposer. This is not the case in Example 19 where player 2 is a veto responder responding before player 3. In this example, player 3 sacrifices a payoff in period 4 in order to have a better position in the TU game that is in force in period 5. The proposer needs player 3 to be non-myopic in period 4 in order to get a payoff higher than the payoff provided in a MBRE outcome. Hence, the proposer could not have achieved a payoff above the MBRE outcome by making balanced proposals.

The next two corollaries are immediate consequences of the results in the previous section.

Corollary 21 Let $(N, v)$ be a veto balanced $T U$ game and $G^{n}(N, v)$ its associated tree game. Let $z=\sum_{1}^{n} x^{t}$ be an outcome resulting from some SPE of the game $G^{n}(N, v)$. If $z$ differs from $\phi(N, v)$ then $z_{1}$ cannot be achieved by making balanced proposals.

If $z$ differs from $\phi(N, v), z_{1} \geq \phi_{1}(N, v)$ (otherwise the proposer would prefer to play the strategy described in lemma 7 ). If the proposer is using 
balanced proposals, he can only achieve at least $\phi_{1}(N, v)$ if all players are getting their serial rule payoffs, that is, if $z=\phi(N, v)$ (corollary 15 and lemma 16). Hence, $z_{1}$ is not achievable by balanced proposals.

Corollary 22 Let $(N, v)$ be a veto balanced $T U$ game and $G^{n}(N, v)$ its associated tree game. Let $z=\sum_{1}^{n} x^{t}$ be an outcome resulting from some SPE of the game $G^{n}(N, v)$. If z differs from $\phi(N, v)$ then there exists at least a stage $t$ and a player $p$ for which $f_{1 p}\left(x^{t},\left(N, v^{t}\right)\right)>x_{p}^{t} \geq 0$.

If $x_{l}^{t} \geq f_{1 l}\left(x^{t}, v^{t}\right)$ for all $l$ and $t, z$ would be achievable under myopic behavior of the responders by proposing $x^{t}$ in each period $t$, but we have established that the only way in which player 1 can get at least $\phi_{1}(N, v)$ under myopic behavior of the responders is if $z=\phi(N, v)$ (lemma 10, corollary 15 and lemma 16).

Note that, if all players behave as myopic maximizers, then for any stage $t$ and any player $p$ it holds that $f_{1 p}\left(x^{t}, v^{t}\right) \leq x_{p}^{t}$. Note also that, given an SPE outcome $z=\sum_{1}^{n} x^{t}$ of the game $G^{n}(N, v)$, if a player $p$ behaves as myopic maximizer in period $t$ then $f_{1 p}\left(x^{t}, v^{t}\right)>x_{p}^{t}$ is possible, if and only if there is a stage in which a responder after player $p$ does not behave myopically.

We denote by $x^{t, i}$ the proposal that emerges in period $t$ immediately after $i$ gets the move. The following lemma establishes a property of $x^{t, i}$ that must be inherited by the final outcome in period $t, x^{t}$.

Lemma 23 Suppose after player $i$ responds to the proposal in period $t$ it holds that $f_{1 i}\left(x^{t, i}, v^{t}\right)>0$. Then $f_{1 i}\left(x^{t}, v^{t}\right)>0$ regardless of the responses of the players moving after $i$.

Proof. Suppose by contradiction that $f_{1 i}\left(x^{t}, v^{t}\right) \leq 0$. This means that at the end of period $t$ there is a coalition $S^{*}$ such that $i \notin S^{*}$ and $v^{t}\left(S^{*}\right) \geq$ $x^{t}\left(S^{*}\right)$. Because $f_{1 i}\left(x^{t, i}, v^{t}\right)>0$ immediately after $i$ responds to the proposal, all coalitions excluding $i$ had a positive satisfaction at that point, and in particular $v^{t}\left(S^{*}\right)<x^{t, i}\left(S^{*}\right)$. There must be a player $h$ moving after $i$ such that $h \notin S^{*}$ and $h$ has received a payoff transfer from player 1 by rejecting the proposal. At the moment of rejection by $h$ we have $f_{1 h}\left(x^{t, h}, v^{t}\right)=x_{h}^{t}>0$. 
However, since $S^{*}$ can be used by 1 to complain against $h$, at the end of period $t$ we have $f_{1 h}\left(x^{t}, v^{t}\right) \leq 0$. There must be another player $l$ moving after $h$ that has received a payoff transfer from player 1, and this player cannot be in $S^{*}$. Then this player is in the same situation as player $h$ : he has $f_{1 l}\left(x^{t, l}, v^{t}\right)>0$ at the moment of rejection, but at the end of period $t$ he has $f_{1 l}\left(x^{t}, v^{t}\right) \leq 0$. Thus there must be another player moving after him... but the number of players is finite.

Notice that lemma 23 holds for any strategy profile, not necessarily an equilibrium.

Lemma 24 Let $(N, v)$ and $(N, w)$ be two veto balanced games in which player 1 is a veto player. Let $w(S) \geq v(S)$ for any $S$. Let $G^{m}(N, v)$ and $G^{m}(N, w)$ be the associated tree games with $m$ proposals. If the payoff provided to the proposer by a SPE outcome of the game $G^{m}(N, w)$ is strictly lower than the payoff provided to the proposer by a SPE outcome of the game $G^{m}(N, v)$, then the SPE outcome of $G^{m}(N, v)$ is such that $x_{l}^{t}<f_{1 l}\left(x^{t}, v^{t}\right)$ for some $l$ and $t$, which implies that at least one responder is behaving nonmyopically.

Proof. Suppose the final payoffs are such that $x_{l}^{t} \geq f_{1 l}\left(x^{t}, v^{t}\right)$ for all $l$ and $t$. We can then use lemma 10 to construct a sequence of balanced proposals with the same payoff for the proposer. For any sequence $y^{t}$ of balanced proposals of the game $G^{m}(N, v)$ it holds that $y_{l}^{t} \geq f_{1 l}\left(y^{t}, w^{t}\right)$, and we can use lemma 10 again to construct a sequence of balanced proposals for the game $G^{m}(N, w)$ where the payoff of the proposer does not change, a contradiction.

Lemma 25 Let $(N, v)$ be a veto balanced TU game. Consider the associated game with $m$ periods $G^{m}(N, v)$. Fix a period $l \in\{1, \ldots, m\}$ and a subgame that starts in period l (not necessarily on the equilibrium path), and label the responders according to the nondecreasing order of d-values in the game $v^{l}$. Let $y^{l}=\sum_{l}^{m} x^{t}$ be the vector of payoffs accumulated between $l$ and $m$. Then $y_{i}^{l} \geq y_{1}^{l}-d_{i}^{l}$ for all $i \in\{2, \ldots, n\}$ in any SPE of $G^{m}(N, v)$. Moreover, the inequality is strict if $d_{i}^{l}>d_{2}^{l}$. 
Proof. Note that, because period $m$ is the last period of the game, myopic and rational behavior coincide, so $x_{i}^{m} \geq f_{1 i}\left(v^{m}, x^{m}\right)$ for all $i$.

Consider player 2. Since players play myopically in period $m$, it must be the case that ${ }^{9}$

$$
\begin{aligned}
x_{2}^{m} & \geq f_{12}\left(x^{m}\right) \geq x_{1}^{m}-\left(d_{2}^{l}-\sum_{l}^{m-1} x_{1}^{t}\right)= \\
& =\sum_{l}^{m} x_{1}^{t}-d_{2}^{l}=y_{1}^{l}-d_{2}^{l} .
\end{aligned}
$$

Since $y_{2}^{l} \geq x_{2}^{m}$, it follows that $y_{2}^{l} \geq y_{1}^{l}-d_{2}^{l}$.

Now consider player $i \neq 2$. There are two possible cases, depending on whether $y_{1}^{l} \leq d_{2}^{l}$.

If $y_{1}^{l} \leq d_{2}^{l}$, the result follows immediately since

$$
y_{1}^{l}-d_{i}^{l} \leq y_{1}^{l}-d_{2}^{l} \leq 0 \leq y_{i}^{l} .
$$

It is also clear that the inequality is strict if $d_{2}^{l}<d_{i}^{l}$.

From now on we assume $y_{1}^{l}>d_{2}^{l}$. Note that since we have already shown that $y_{2}^{l} \geq y_{1}^{l}-d_{2}^{l}$, it follows that that $y_{2}^{l}>0$ in this case. There are again two possible cases, depending on whether the coalition associated to $f_{1 i}\left(x^{m}, v^{m}\right)$ contains 2 .

If the coalition contains 2 , we have

$$
\begin{aligned}
y_{i}^{l} & \geq x_{i}^{m} \geq f_{1 i}\left(x^{m}, v^{m}\right) \geq x_{1}^{m}+x_{2}^{m}-\left(d_{i}^{l}-\sum_{l}^{m-1} x_{1}^{t}-\sum_{l}^{m-1} x_{2}^{t}\right)= \\
& =y_{1}^{l}+y_{2}^{l}-d_{i}^{l}>y_{1}^{l}-d_{i}^{l},
\end{aligned}
$$

where the last inequality follows from the fact that $y_{2}^{l}>0$.

If the coalition does not contain 2, we have $f_{1 i}\left(x^{m}\right) \geq f_{12}\left(x^{m}\right)$. Then

$$
y_{i}^{l} \geq x_{i}^{m} \geq f_{1 i}\left(x^{m}, v^{m}\right) \geq f_{12}\left(x^{m}, v^{m}\right) \geq y_{1}^{l}-d_{2}^{l} \geq y_{1}^{l}-d_{i}^{l} .
$$

\footnotetext{
${ }^{9}$ If $S$ is a coalition associated to $f_{12}\left(x^{n}\right)$, the total payoff of $S$ must be at least $x_{1}^{n}$. Also, the total value of $S$ must be at most $d_{2}^{l}-\sum_{l}^{n-1} x_{1}^{t}$.
} 
Note that the inequality is strict for $d_{i}^{l}>d_{2}^{l}$.

Next lemma shows that the outcome of any SPE coincides with the outcome obtained by using a profile of strategies where all responders behave as myopic maximizers.

Lemma 26 Let $(N, v)$ be a veto balanced TU game. Consider the associated game $G^{m}(N, v)$ in which the responders move following the order of nonincreasing $d$-values of $v^{k}$. Let $z=\sum_{1}^{m} x^{t}$ be an outcome resulting from some SPE of the game $G^{m}(N, v)$. Then the proposer can obtain $z_{1}$ by making balanced proposals.

Proof. Suppose on the contrary that $z_{1}$ cannot be obtained with balanced proposals. By Lemma 10 we know that there is a player $k$ and a stage $t$ such that $f_{1 k}\left(x^{t}, v^{t}\right)>x_{k}^{t} \geq 0$; otherwise the proposer can obtain $z_{1}$ with balanced proposals.

Let $t$ be the last period ${ }^{10}$ in which for some responder it holds that $f_{1 k}\left(x^{t}, v^{t}\right)>x_{k}^{t} \geq 0$. Let $k$ be the last responder at $t$ for whom $f_{1 k}\left(x^{t}, v^{t}\right)>$ $x_{k}^{t} \geq 0$. We consider two cases:

a) There is a player $p$ with $d_{p}^{t} \leq d_{k}^{t}$ such that $f_{1 p}\left(x^{t}, v^{t}\right) \leq 0$. Note that $f_{1 k}\left(x^{t}, v^{t}\right)>x_{k}^{t} \geq 0$ means that any coalition without player $k$ has a positive satisfaction and, in particular any coalition $S_{k} \in$ $\arg \max _{S \subseteq N \backslash\{k\}} v^{t}(S)$. On the other hand since $f_{1 p}\left(x^{t}, v^{t}\right) \leq 0$ then there exists a coalition without player $p$ for which the satisfaction is not positive. Let $S_{p}^{*}$ be one such coalition (it must contain $k$ ). Then we have the following two inequalities:

$$
x^{t}\left(S_{k}\right)>d_{k}^{t} \text { and } v^{t}\left(S_{p}^{*}\right) \geq x^{t}\left(S_{p}^{*}\right)
$$

Combining the two inequalities we obtain

$$
x^{t}\left(S_{k}\right)-x^{t}\left(S_{p}^{*}\right)>d_{k}^{t}-v^{t}\left(S_{p}^{*}\right) \geq d_{k}^{t}-d_{p}^{t} \geq 0 .
$$

\footnotetext{
${ }^{10}$ It is clear that $t<m$, since all responders behave as myopic maximizers in the last period.
} 
The inequality above implies that there are players not in $S_{p}^{*}$ receiving a positive payoff in period $t$.

Consider a new allocation, $y^{t}$, which is identical to $x^{t}$ except that $y_{i}^{t}=0$ for all $i$ not in $S_{p}^{*}$ (thus $y_{i}^{t}=x_{i}^{t}$ for all $i$ in $S_{p}^{*}$ ). We now show that $f_{1 i}\left(y^{t}, v^{t}\right) \leq y_{i}^{t}$ for all $i \in N$, so that player 1 can get the same payoff with balanced proposals by lemma 10 .

For any player $i$ it holds that $f_{1 i}\left(y^{t}, v^{t}\right) \leq f_{1 i}\left(x^{t}, v^{t}\right)$.

Because $S_{p}^{*}$ can be used against any player outside $S_{p}^{*}$, for any player outside $S_{p}^{*}$ it holds that $f_{1 i}\left(y^{t}, v^{t}\right) \leq f_{1 p}\left(y^{t}, v^{t}\right) \leq f_{1 p}\left(x^{t}, v^{t}\right) \leq 0$. Thus, $f_{1 i}\left(y^{t}, v^{t}\right) \leq y_{i}^{t}$ for all $i \notin S_{p}^{*}$.

Can there be a player $l \in S_{p}^{*}$ for which $f_{1 l}\left(y^{t}, v^{t}\right)>y_{l}^{t}$ ? If so, this inequality must have existed already for $x^{t}$, since $f_{1 l}\left(y^{t}, v^{t}\right) \leq f_{1 l}\left(x^{t}, v^{t}\right)$ and $y_{l}^{t}=x_{l}^{t}$. Since player $k$ is the last player satisfying the inequality for $x^{t}$, it must be the case that $d_{l}^{t} \geq d_{k}^{t} \geq d_{p}^{t}$, thus we can repeat the reasoning above with $S_{l}$ and $S_{p}^{*}$ and, given that nothing has changed for $S_{p}^{*}$, we would conclude that $y^{t}\left(S_{l}\right)-y^{t}\left(S_{p}^{*}\right)>0$, a contradiction since all players outside $S_{p}^{*}$ have zero payoffs. Thus, $f_{1 i}\left(y^{t}, v^{t}\right) \leq y_{i}^{t}$ for all $i$.

Note that for this part of the proof no assumption is needed about the order in which the responders move. ${ }^{11}$

b) The second case is $f_{1 l}\left(x^{t}, v^{t}\right)>0$ for all players moving after $k$ at $t$. By assumption, on the equilibrium path from $t+1$ onwards all proposals have an associated balanced proposal. We distinguish two subcases:

b1) The last player to act nonmyopically at $t$ has accepted a proposal. This player must be player $k$ or a player moving after $k$. Call this player $p$ ( $p$ moving after $k$ is possible if a myopic rejection by a player moving after $p$ has restored $f_{1 p} \leq x_{p}$ ).

We will show that it is not in $p$ 's interest to accept the proposal. To do this, we need to analyze two subgames: the subgame on

\footnotetext{
${ }^{11}$ All we need to assume in this part of the proof is that player $k$ is the "last" player in the sense of being the player with the lowest $d_{i}^{t}$, not necessarily the one who moves last.
} 
the equilibrium path in which $p$ accepts the proposal, and the subgame off the equilibrium path in which $p$ rejects the proposal. We will talk about the $A$-path (the equilibrium path) and the $R$ path. Denote by $x_{A}^{t}$ and $x_{R}^{t}$ the final payoffs at stage $t$ depending on whether player $p$ accepts or rejects the proposal. If player $p$ rejects the proposal, we take any subgame perfect equilibrium of that subgame. Denote by $v_{A}^{t+1}$ and $v_{R}^{t+1}$ the corresponding TU games at $t+1$.

Because $f_{1 l}\left(x^{t}, v^{t}\right)>0$ for $l \in\{2, \ldots, p\}$, on the $A$-path all players in $\{2, \ldots, p\}$ are veto players at $t+1$.

The game $v_{R}^{t+1}$ is better than the game $v_{A}^{t+1}$ (in the sense of lemma 24). If $v_{A}^{t+1}(S)>0$, coalition $S$ must contain all players in $\{1,2, \ldots, p\}$. For this kind of coalition $v_{R}^{t+1}(S)=v_{A}^{t+1}(S)$, since any payoff transfers after rejection occur between members of $\{1, \ldots, p\}$ (here the order of moves is essential). Thus, $v_{A}^{t+1}(S) \leq v_{R}^{t+1}(S)$ for all $S$.

We now show that $p$ is also veto at $t+1$ on the $R$-path.

Suppose $p$ is not veto at $t+1$ on the $R$-path. Then there is a coalition $S_{p}$ such that $p \notin S_{p}$ and $v^{R, t+1}\left(S_{p}\right)>0$. This can only happen if $v^{R, t}\left(S_{p}\right)>x_{R}^{t}\left(S_{p}\right)$, or equivalently $f_{1 p}\left(x_{R}^{t}, v^{t}\right)<0$, contradicting lemma 23.

Thus, player $p$ is a veto player at $t+1$ regardless of whether he accepts or rejects the proposal. Given the order of moves, veto players can secure at least the same payoff as the proposer. There is no reason for veto players to act nonmyopically because the game at $t+2$ will be the same regardless of how the payoff is distributed at $t+1$ between veto players; no payoff can go to anyone else given the order of responders. For the same reason the proposer will never make a proposal that gives another veto player more than he gets himself, so that all veto players must get the same payoff given the order of moves. Let $y_{1}^{R}$ be player 1 's payoff if $p$ rejects the proposal (this is the payoff accumulated between periods $t+1$ and $n$ ) and $y_{1}^{A}$ be player 1 's payoff if $p$ accepts 
the proposal. Because of lemma 24, the only way in which $y_{1}^{A}$ can exceed $y_{1}^{R}$ is if there is a nonmyopic move at $v_{A}^{t+1}$ that leads to $f_{1 i}\left(x_{A}^{t+1}, v_{A}^{t+1}\right)>x_{i}^{A, t+1}$ for some $i$. By assumption this is not the case. Thus, it was not in $p$ 's interest to accept: rejecting would yield a higher payoff at $t$, and at least the same payoff in the rest of the game.

b2) The last player to act nonmyopically at $t$ has rejected a proposal. Let $p$ be the last player to act nonmyopically at $t$. This player cannot be player $k$ because after any rejection (myopic or otherwise) it holds that $f_{1 k}(.) \leq x_{k}^{t}$, and given that the remaining responders act myopically this inequality would never be reversed. Someone moving after $k$ must have rejected a proposal nonmyopically (transferring payoff to the proposer) and created the inequality $f_{1 k}\left(x^{t}, v^{t}\right)>x_{k}^{t}$, hence player $p$ must be moving after $k$. We will show that it is not in $p$ 's interest to reject the proposal. To do this, we need to analyze two subgames: the subgame on the equilibrium path in which $p$ rejects the proposal, and the subgame off the equilibrium path in which $p$ accepts the proposal. We will talk about the $R$-path (the equilibrium path) and the $A$-path.

Because $f_{1 l}\left(x^{t}, v^{t}\right)>0$ for $l \in\{2, \ldots, k\}$, on the equilibrium path all players in $\{2, \ldots, k\}$ are veto players at $t+1$.

The game $v_{A}^{t+1}$ is better than the game $v_{R}^{t+1}$ (in the sense of lemma 24). If $v_{R}^{t+1}(S)>0$, coalition $S$ must contain all players in $\{1,2, \ldots, p\}$. For this kind of coalition $v_{R}^{t+1}(S)=v_{A}^{t+1}(S)$, since any payoff transfers on the $A$-path must occur between members of $\{1,2, \ldots, p\}$ (again, here the order of moves is essential). Thus, $v_{R}^{t+1}(S) \leq v_{A}^{t+1}(S)$ for all $S$.

Suppose player $p$ is veto also on the $A$-path. Then the reasoning of case b1 applies, and there is no reason for $p$ to act nonmyopically in period $t$.

Now suppose player $p$ is not veto on the $A$-path at $t+1$. We define $d_{A p}^{t+1}:=\max _{S \subseteq N \backslash\{p\}} v_{A}^{t+1}(S)$. Since $p$ is not a veto player at 
$t+1$ on the $A$-path, $d_{A p}^{t+1}>0$. There is a coalition $S_{p}$ such that $v^{t}\left(S_{p}\right)-x_{A}^{t}\left(S_{p}\right)=d_{A p}^{t+1}>0$. Since by assumption $f_{1 p}\left(x^{t}, v^{t}\right)>0$ on the $R$-path, we also have $x_{R}^{t}\left(S_{p}\right)-v^{t}\left(S_{p}\right)>0$. From the two inequalities we get $x_{R}^{t}\left(S_{p}\right)-x_{A}^{t}\left(S_{p}\right)>d_{A p}^{t+1}$.

Let $\alpha$ be the payoff player $p$ transfers to player 1 when rejecting the proposal (part of this payoff may then go to other players between 2 and $p-1$ if they myopically reject a proposal). We want to show that $\alpha \geq x_{R}^{t}\left(S_{p}\right)-x_{A}^{t}\left(S_{p}\right)$, which implies $\alpha>d_{A p}^{t+1}$. This is not completely obvious because part of the difference could be due to a player outside $S_{p}$ myopically rejecting on the $A$-path. Claim. $\alpha \geq x_{R}^{t}\left(S_{p}\right)-x_{A}^{t}\left(S_{p}\right)>d_{A p}^{t+1}$.

Note that $x_{R}^{t}$ and $x_{A}^{t}$ are identical for all responders moving before $p$. Because of the order of moves, any payoff transfers due to a change of $p$ 's action from $\mathrm{R}$ to A occur within the set $\{1,2, \ldots, p\}$. Denote by $T$ the set $\{2, \ldots, p-1\} \backslash S_{p}$. We can write $x_{R}^{t}\left(S_{p}\right)+$ $x_{R}^{t}(T)=x_{A}^{t}\left(S_{p}\right)+x_{A}^{t}(T)+\alpha$. All we need to show is that $x_{R h}^{t} \geq$ $x_{A h}^{t}$ for all $h \in T$ (this is obvious if $T$ is empty). This implies $x_{R}^{t}(T) \geq x_{A}^{t}(T)$ and hence $x_{R}^{t}\left(S_{p}\right)-x_{A}^{t}\left(S_{p}\right) \leq \alpha$.

Suppose there is a player $h$ in $T$ that has $x_{R h}^{t}<x_{A h}^{t}$. Since this player is not in $S_{p}, S_{p}$ can be used by player 1 against him.

Player $h$ must have rejected in the $A$-path (if he had accepted he would have $\left.x_{R h}^{t} \geq x_{A h}^{t}\right)^{12}$. After rejecting he is left with $f_{1 h}()=.x_{A h}^{t}>0$. On the other hand, $v^{t}\left(S_{p}\right)-x_{A}^{t}\left(S_{p}\right)>0$. Thus, $f_{1 h}\left(x_{A}^{t}, v^{t}\right)<0$, contradicting lemma 23 .

Now we are in a position to compare payoffs on the $A$ and $R$-paths and see that $p$ prefers to accept the proposal.

Since player $p$ is veto on the $R$-path, he gets $y_{1}^{R}$. On the $A$-path, he gets $y_{p}^{A}$, whereas the proposer gets $y_{1}^{A}$. Because lemma 25 applies

\footnotetext{
${ }^{12}$ By assumption, $p$ is the last player to behave nonmyopically on the R-path. This leaves us with two possibilities for $h$ on the R-path: myopic acceptance and myopic rejection. In neither case it is possible for $h$ to get a higher payoff by accepting the proposal on the A-path.
} 
to all subgames regardless of whether they are on the equilibrium path, $y_{p}^{A} \geq y_{1}^{A}-d_{A p}^{t+1}$.

In order to have an equilibrium, $p$ must prefer to reject the proposal, thus we need $x_{p}^{t}+y_{1}^{R} \geq x_{p}^{t}+\alpha+y_{p}^{A}>x_{p}^{t}+d_{A p}^{t+1}+y_{1}^{A}-d_{A p}^{t+1}$. Therefore we need $y_{1}^{R}>y_{1}^{A}$. Since the game $v_{A}^{t+1}$ is better than the game $v_{R}^{t+1}$ (in the sense of lemma 24), and by assumption $f_{1 i}\left(x^{l}, v^{l}\right)>x_{i}^{l}$ never happens on the $R$-path from $t+1$ onwards, the inequality $y_{1}^{R}>y_{1}^{A}$ cannot hold.

We have shown that any SPE outcome is such that the proposer can always achieve $z_{1}$ with balanced proposals. Balanced proposals in the proof were always constructed by (possibly) cutting down some of the responders'

payoffs, never by increasing them. Hence, any SPE outcome, $\sum_{1}^{m} x^{t}$, could be obtained as follows: The proposer proposes sequentially $\left(x^{1}, x^{2}, \ldots, x^{m}\right)$ and the responders behave as myopic maximizers. Therefore, any SPE outcome coincides with the outcome resulting from a profile of strategies where all responders play as myopic maximizers. And among all these profiles, if the proposer plays optimally the outcome is unique (Theorem 17). Therefore,

Theorem 27 Let $(N, v)$ be a veto balanced $T U$ game and $G^{n}(N, v)$ its associated tree game in which the responders move following the order of nonincreasing d-values of $v^{k}$. Then $\phi(N, v)$ is the outcome of any SPE.

\section{Concluding remarks}

We have provided noncooperative foundations for the serial rule $\phi(N, v)$ in veto balanced games. We have also shown that any SPE outcome of our bargaining procedure is achievable with myopic behavior of the responders if responders move by increasing strength (lemma 26). This result is independent of the number of periods. If there are at least $n-1$ periods, the only SPE outcome is the serial rule: the proposer is always able to obtain $\phi_{1}(N, v)$ 
by making balanced proposals, and the only way to obtain this payoff is if all other players get $\phi_{i}(N, v)$ as well. If there are fewer than $n-1$ periods, it is not necessarily possible for the proposer to achieve $\phi_{1}(N, v)$. If $z$ is a SPE outcome, it is still true that the proposer can obtain $z_{1}$ by making balanced proposals, hence all SPE outcomes must have the same $z_{1}$, but there may be several SPE outcomes if $z_{1}<\phi_{1}(N, v)$.

\section{Acknowledgements}

We thank Jeroen Kuipers for his helpful comments. The first author also acknowledges financial support provided by the Project 9/UPV00031.32115352/2003 of University of the Basque Country and the Project BEC200308182 of the Ministry of Education and Science of Spain. This author is also grateful for the hospitality provided by the Department of Economics of the University of Rochester and the financial support provided by the Program "Salvador de Madariaga" of the Ministry of Education and Science of Spain.

\section{References}

[1] Arin J and Feltkamp V (1997) The nucleolus and kernel of veto-rich transferable utility games. Int J of Game Theory 26: 61-73.

[2] Arin J and Feltkamp V (2007) Coalitional games with veto players: consistency, monotonicity and Nash outcomes. Journal of Mathematical Economics 43: 855-870.

[3] Arin J and Feltkamp V (2012) Coalitional games: monotonicity and core. European Journal of Operational Research 216: 208-213.

[4] Dagan N., Serrano R and Volij O (1997) A non-cooperative view of consistent bankruptcy rules. Games and Economic Behavior 18: 55-72.

[5] Davis M and Maschler M (1965) The kernel of a cooperative game. Naval Research Logistics Quarterly 12: 223-259. 
[6] Maschler M (1992) The bargaining set, kernel and nucleolus. Handbook of game theory with economic applications I, Aumann R. J. and Hart S. eds., Amsterdam: North-Holland.

[7] Meggido N (1974) On the nonmonotonicity of the bargaining set, the kernel and the nucleolus of a game. SIAM J of Applied Mathematics 27: 355-358.

[8] Moulin H. and Shenker S (1992) Serial cost sharing. Econometrica 60: 1009-37.

[9] Peleg B (1986) On the reduced game property and its converse. Int J of Game Theory 15: 187-200.

[10] Peleg B and Sudhölter P (2003) Introduction to the theory of cooperative games. Kluwer Academic Publishers.

[11] Schmeidler D (1969) The nucleolus of a characteristic function game. SIAM J of Applied Mathematics 17: 1163-117. 